\title{
Completion rate of physician orders for life- sustaining treatment for patients with metastatic or recurrent cancer: a preliminary, cross-sectional study
}

Ju Won Kim¹, Jung Yoon Choi ${ }^{1}$, Won Jin Jang ${ }^{1}$, Yoon Ji Choi ${ }^{1}$, Youn Seon Choi ${ }^{2}$, Sang Won Shin ${ }^{1}$, Yeul Hong Kim ${ }^{1}$ and Kyong Hwa Park ${ }^{1 *}$ (D)

\begin{abstract}
Background: "End of life" is a difficult topic of conversation in East Asian cultures, even among patients and doctors who share a good rapport. In 2016, the Hospice, Palliative Care, and Life-Sustaining Treatment DecisionMaking Act, which took the form of "Physician Orders for Life-Sustaining Treatment," was introduced in South Korea. This study was conducted to investigate the completion rate of Physician Orders for Life-Sustaining Treatment in patients with advanced cancer on the active recommendation of physicians, as well as patients' general attitudes toward end-of-life care.

Methods: We conducted a preliminary, cross-sectional descriptive survey on patients with advanced cancer. A total of 101 patients with advanced solid cancer agreed to participate in the study. The primary endpoint was the rate of completion of Physician Orders for Life-Sustaining Treatment based on a doctor's suggestion. Written interviews were conducted to understand the perceptions and factors influencing patients' decisions.

Results: Of the 101 patients, 72 (71.3\%) agreed to prepare Physician Orders for Life-Sustaining Treatment. Patients who had an educational level of high school or higher were more likely to agree to complete Physician Orders for Life-Sustaining Treatment documentation as compared to the lower educational status group. More than half of the respondents who completed Physician Orders for Life-Sustaining Treatment documentation reported that they had more than a fair understanding of "life-sustaining care" or "Physician Orders for Life-Sustaining Treatment." Participants' reasons for Physician Orders for Life-Sustaining Treatment completion were diverse.

Conclusions: We found that highly educated patients, who understood the concept behind the policy well, tended to accept Physician Orders for Life-Sustaining Treatment without hesitation. Better education, information shared through the media, and conversations with health care providers might improve understanding of Physician Orders for Life-Sustaining Treatment in patients with cancer.
\end{abstract}

Keywords: Physician orders for life-sustaining treatment, End-of-life care, Advance directives

\footnotetext{
* Correspondence: khpark@korea.ac.kr

${ }^{1}$ Division of Oncology/Hematology, Department of Internal medicine, Korea University Anam Hospital, 73 Goryeodae-ro Seongbuk-gu, Seoul 02841, South Korea

Full list of author information is available at the end of the article
}

(c) The Author(s). 2019 Open Access This article is distributed under the terms of the Creative Commons Attribution 4.0 International License (http://creativecommons.org/licenses/by/4.0/), which permits unrestricted use, distribution, and reproduction in any medium, provided you give appropriate credit to the original author(s) and the source, provide a link to the Creative Commons license, and indicate if changes were made. The Creative Commons Public Domain Dedication waiver (http://creativecommons.org/publicdomain/zero/1.0/) applies to the data made available in this article, unless otherwise stated. 


\section{Background}

Talking about death and dying is one of the key steps in developing an advance care plan (ACP) for patients with cancer. However, end of life (EOL) is a sensitive topic of conversation even between patients and health care providers who share a good rapport. In addition to the sensitivity of the topic, the appropriate process and timing are controversial, making conflicts in the medical field inevitable.

In South Korea, as the demand for establishing a legal basis for the EOL decision-making process increased, the Hospice, Palliative Care, and Life-Sustaining Treatment Decision-Making Act passed the plenary session of the National Assembly in January 2016. After a threemonth pilot period, the Act was fully implemented on February 4, 2018. Under this Act, those who meet the requirements can state their intentions regarding lifesustaining medical care through the Physician Orders for Life-Sustaining Treatment (POLST) document [1]. POLST is a part of an ACP with advance directives (AD) and is written by a doctor based on the patient's wishes at the terminal stage (Additional file 2: Figure S1).

Along with geriatric chronic diseases, cancer is cited as a major cause of mortality in South Korea. This is because almost all kinds of cancer ultimately cause multi-organ failure with metastases, which is often difficult to revive in spite of active treatment. Along with the context that there is lack of palliative care specialist in South Korea, the policy around ACT was discussed mainly among physicians who treat cancers. Moreover, medical oncologists were the most important instructor to patients in the clinic.

Nevertheless, the sociocultural situation in East Asian countries such as South Korea is still such that discussions of ACP, including EOL, tend to be uncomfortable $[2,3]$. Additionally, proposals to prepare for dying well are still taboo. Decisions regarding the dying process are more likely to be a reflection of complex familial dynamics than the patient's free will [4]. To achieve the purpose of the legislation, it is essential that not only medical staff and caregivers but also patients themselves be fully aware of ACP policies.

However, there are no studies on the real-world barriers to EOL care and POLST decision-making among patients with cancer, and there is a lack of nationwide surveys on patients' perceptions. We, therefore, conducted this study to investigate the completion rate of POLST in patients with advanced cancer when it is actively suggested by their physicians, and to examine patients' general attitudes toward EOL.

\section{Methods}

\section{Study protocol}

This was a single-center, preliminary, cross-sectional study conducted from June 2018 to January 2019 at the
Korea University Anam Hospital Cancer Center. Patients were offered POLST at physicians' discretion if they were considered to be nearing EOL based on their medical progress. The doctors defined eligible patients based on remnant organ function, poor general condition, which was defined as Eastern Cooperation Oncology Group grade 3 or 4, and the lack of available treatment choice. Three medical oncologists directly provided an outline of POLST to their patients who were eligible for the study. After a thorough discussion, patients were asked to decide whether they would prepare the document that day. After they had made their decision, patients were asked to complete questionnaires in a protected space for up to $30 \mathrm{~min}$, regardless of whether or not they decided to complete POLST documentation. Participants filled out and submitted the questionnaire on the spot. Written informed consent was obtained from all participants and the study was approved by the Institutional Review Board (IRB) of Korea University Hospital (IRB no. 2018AN0152).

\section{Study participants}

This study aimed to investigate the POLST completion rate in patients with recurrent or metastatic cancer who were currently undergoing treatment. Only patients over 19 years of age who could understand the purpose of the study and provide informed consent were included. Patients who could not understand the Korean questionnaire were excluded. There was no distinction made on the basis of type of cancer.

\section{POLST in South Korea and survey questionnaire}

In South Korea, the Hospice, Palliative Care, and LifeSustaining Treatment Decision-Making Act introduced AD and POLST as legal forms of ACP in 2016. The South Korean POLST documentation contains basic information on the patient and doctor and an indication of the decision to refuse life-sustaining treatment. Cardiopulmonary resuscitation (CPR), artificial respiration through tracheal intubation, hemodialysis, and anti-cancer therapy are represented as optional life-sustaining medical services, and patients can choose whether or not to implement any of them in their last days of life. As a part of ACP, the POLST document may also include choice of hospice palliative care. After the patient has confirmed their choice by signing the document, it is registered in the central government system and preserved for 10 years.

We conducted a survey questionnaire to identify factors associated with the completion rate of POLST. The questionnaires were reviewed and confirmed by multiple medical staff members, including medical oncologists and family physicians. The survey questionnaire requested the following: (1) demographic information: age, gender, religion, level of education, and monthly income; 
(2) self-reported level of understanding of life-sustaining treatment and POLST (very familiar, familiar, fair understanding, not familiar, never heard of it); (3) major sources of information on life-sustaining treatment and POLST; (4) reason for choosing (or refusing) to complete POLST documentation; (5) person with whom they discussed their EOL care; (6) optimal timing for discussing ACP; (7) ideal place to meet their EOL; and (8) whether or not they wanted to avail the services of a hospice care center and why.

\section{Statistical analysis}

The primary endpoint of this study was the POLST completion rate in patients with metastatic or recurrent cancer who were undergoing active anti-cancer treatment. Secondary outcomes were factors related to POLST completion rate and various aspects of practical EOL care.

If the questionnaire was not answered completely, only the collected data were analyzed. The questions that were not responded to were treated as missing data, and no specific alternative was used. Multiple responses were allowed depending on the type of question, and these were calculated separately for each question.

The chi-square test was used to examine associations between patients' demographic characteristics, degree of awareness of the system, and POLST completion. All reported $p$-values were two sided, and $\mathrm{p}$-values $<0.05$ were considered significant. Statistical analysis was conducted using SPSS version 24.0.

\section{Results}

\section{Study population}

A total of 119 patients were offered POLST, and 101 were eligible to participate in this study; a schematic depiction of the study population is presented in Fig. 1. The return rate of the questionnaires was $100 \%$, and an analysis was conducted based on the patients' responses. The demographic characteristics of the 101 participants are summarized in Table 1. The median age was 64 years (range 30-90 years), and the proportion of male and female participants was similar (male 53.5\%, female $46.5 \%$ ). Breast cancer was the most common type of cancer $(33,32.7 \%)$, followed by prostate cancer (22, $21.8 \%)$. Median time from cancer diagnosis to suggestion of POLST was 30.8 months (range 1.8-262.5), and median follow-up period by the physician who suggested POLST was 16.8 months (range 1.2-106.9). Most of the patients participated in the study during their visit to the outpatient clinic (93.1\%).

\section{POLST completion rate and reason for the decision}

Of the 101 patients, 72 (71.3\%) agreed to sign the POLST form and registered the document on the same day. Out of the 72 patients who completed POLST documentation, 49 (67.1\%) excluded CPR, artificial respiration, and hemodialysis, but wanted to continue with chemotherapy. Further, 21 patients (29.2\%) did not want any kind of lifesustaining treatment, and two patients $(2.8 \%)$ who were in the fifth stage of chronic kidney disease at the time of signing the POLST form wanted to suspend only CPR and artificial respiration. In total, CPR and artificial respiration were declined by all participants who prepared POLST,

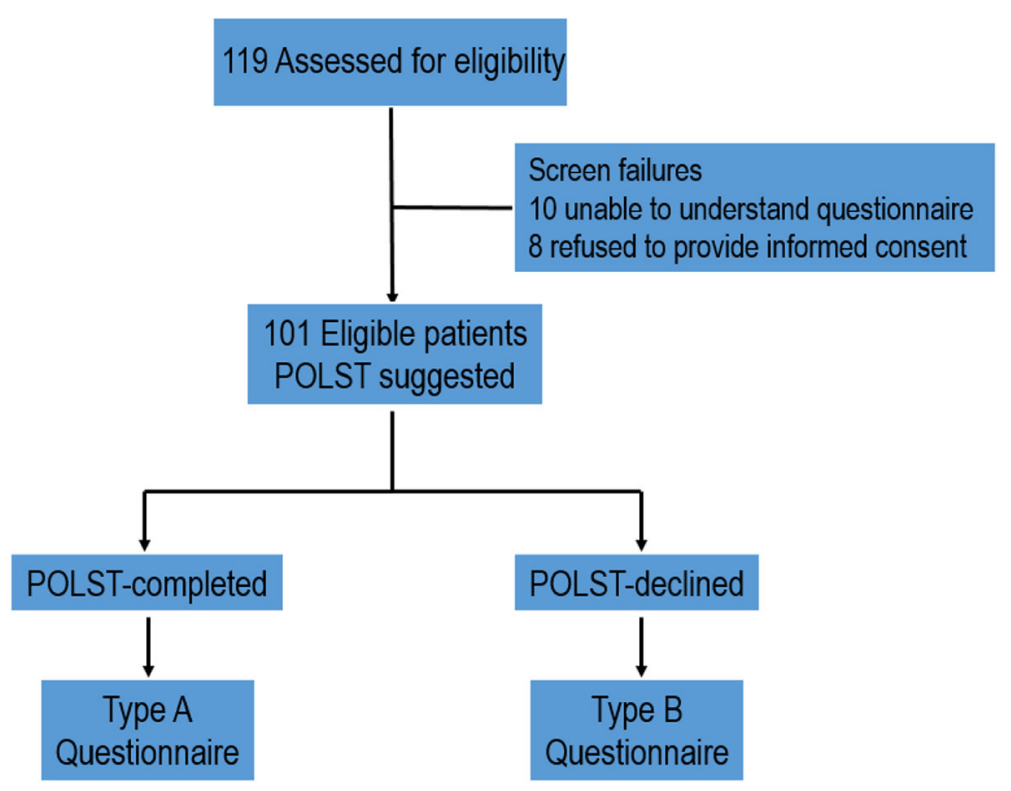

Fig. 1 Schematic representation of the study population 
Table 1 Demographic features of the study population $(N=101)$

\begin{tabular}{|c|c|}
\hline Characteristics & No. (\%) \\
\hline \multicolumn{2}{|l|}{ Age (years) } \\
\hline $31-40$ & $3(3.0 \%)$ \\
\hline $41-50$ & $11(10.9 \%)$ \\
\hline $51-60$ & $24(23.8 \%)$ \\
\hline $61-70$ & $29(28.7 \%)$ \\
\hline $71-80$ & $27(26.7 \%)$ \\
\hline $81-90$ & $7(6.9 \%)$ \\
\hline \multicolumn{2}{|l|}{ Gender } \\
\hline Male & 47 (46.5\%) \\
\hline Female & $54(53.5 \%)$ \\
\hline \multicolumn{2}{|l|}{ Cancer type } \\
\hline Breast & $33(32.7 \%)$ \\
\hline Prostate & $22(21.8 \%)$ \\
\hline Lung & $2(2.0 \%)$ \\
\hline Gastrointestinal & $27(26.7 \%)$ \\
\hline $\begin{array}{l}\text { Genitourinary } \\
\text { (except prostate) }\end{array}$ & $8(7.9 \%)$ \\
\hline Hepatobiliary & $5(5.0 \%)$ \\
\hline Others $^{a}$ & $4(4.0 \%)$ \\
\hline \multicolumn{2}{|l|}{ Religion } \\
\hline No religion & $43(42.6 \%)$ \\
\hline Follow a religion ${ }^{b}$ & 57 (56.4\%) \\
\hline Unknown & $1(1.0 \%)$ \\
\hline \multicolumn{2}{|l|}{ Educational status } \\
\hline High school or below & $40(39.6 \%)$ \\
\hline High school or above & $61(60.4 \%)$ \\
\hline \multicolumn{2}{|l|}{ Monthly income } \\
\hline Less than $\$ 2000$ & $77(76.2 \%)$ \\
\hline More than $\$ 2000$ & $20(19.8 \%)$ \\
\hline Unknown & $4(4.0 \%)$ \\
\hline \multicolumn{2}{|l|}{$\begin{array}{l}\text { Place of } \\
\text { POLST suggestion }\end{array}$} \\
\hline Outpatient clinic & $94(93.1 \%)$ \\
\hline General ward & $7(6.9 \%)$ \\
\hline
\end{tabular}

a Others: 3 sarcomas, 1 melanoma

b 21 Christianity, 8 Catholicism, 23 Buddhism, 5 Other

and hemodialysis was refused by $97.2 \%$. However, the suspension of chemotherapy was requested by only $31.9 \%$ of patients. The questionnaires provided to each group presented four choices for the reason they decided (not) to prepare POLST. The results and the proportion of each answer are visualized in Fig. 2. The most common reason for preparing POLST was "to exercise my own will, not that of my caregiver" $(28,38.9 \%)$, followed by "because my doctor recommended it" (25, 34.7\%). The two major reasons for refusal were "need to discuss it further with family" $(11,37.9 \%)$ and "need more time by myself to think about it" $(8,27.6 \%)$.

\section{POLST completion rate according to demographic features}

We compared POLST completion rate between two groups stratified by demographic features. We divided patients according to their age and gender, educational status, monthly income, and whether or not they followed a religion, and then analyzed which group tended to complete POLST documentation. Mean or median values were used as the basis for dividing the two groups. Table 2 summarizes the POLST completion rate according to demographic and socioeconomic features. According to the results, younger $(73.1 \%$ vs. $69.4 \%, p=0.626)$ female (72.3\% vs. $70.4 \%, p=1$ ) patients who did not follow a religion $(72.1 \%$ vs. $70.2 \%, \mathrm{p}=1)$ and had a high educational level $(82.0 \%$ vs. $56.4 \%, p=0.007)$ and higher monthly income $(85.0 \%$ vs. $66.2 \%, p=0.169)$ showed the highest rate of POLST completion. None of the differences in POLST completion rate between the groups were significant except for educational status.

\section{Comparison of awareness between POLST-completion and non-completion groups}

The perception of life-sustaining treatment and POLST was investigated, and the results are summarized in Table 3 . The understanding of both concepts was higher in the POLSTcompletion group, and this was statistically significant (average "EOL care" understanding score 3.32 vs. 2.68, $p=0.016$ and "POLST" understanding score 2.99 vs. 2.34, $p=0.036$ ). In both groups, conventional media like newspapers and television were the main source of information about EOL, followed by medical staff (50.0 and 31.9\% in the completion group, and 48.3 and $20.7 \%$ in the non-completion group, respectively).

\section{Questions about EOL care}

Participants were asked three questions regardless of whether or not they decided to complete POLST documentation (Additional file 1: Table S1). The decision regarding ACP was mainly taken by the patients themselves (51, 50.5\%), followed by the spouse $(21,20.8 \%)$ and physicians $(18,17.8 \%)$. Almost half the participants chose "when someone is young and healthy" $(50,49.5 \%)$ as the optimal time for preparing POLST. The most suitable places to meet their EOL were "home" $(35,34.7 \%)$ and "university hospitals" (24, 23.8\%).

\section{Willingness to use a hospice care center}

The South Korean POLST recommends indicating one's willingness to use a hospice care center. The present survey also asked about this and collected the reasons 


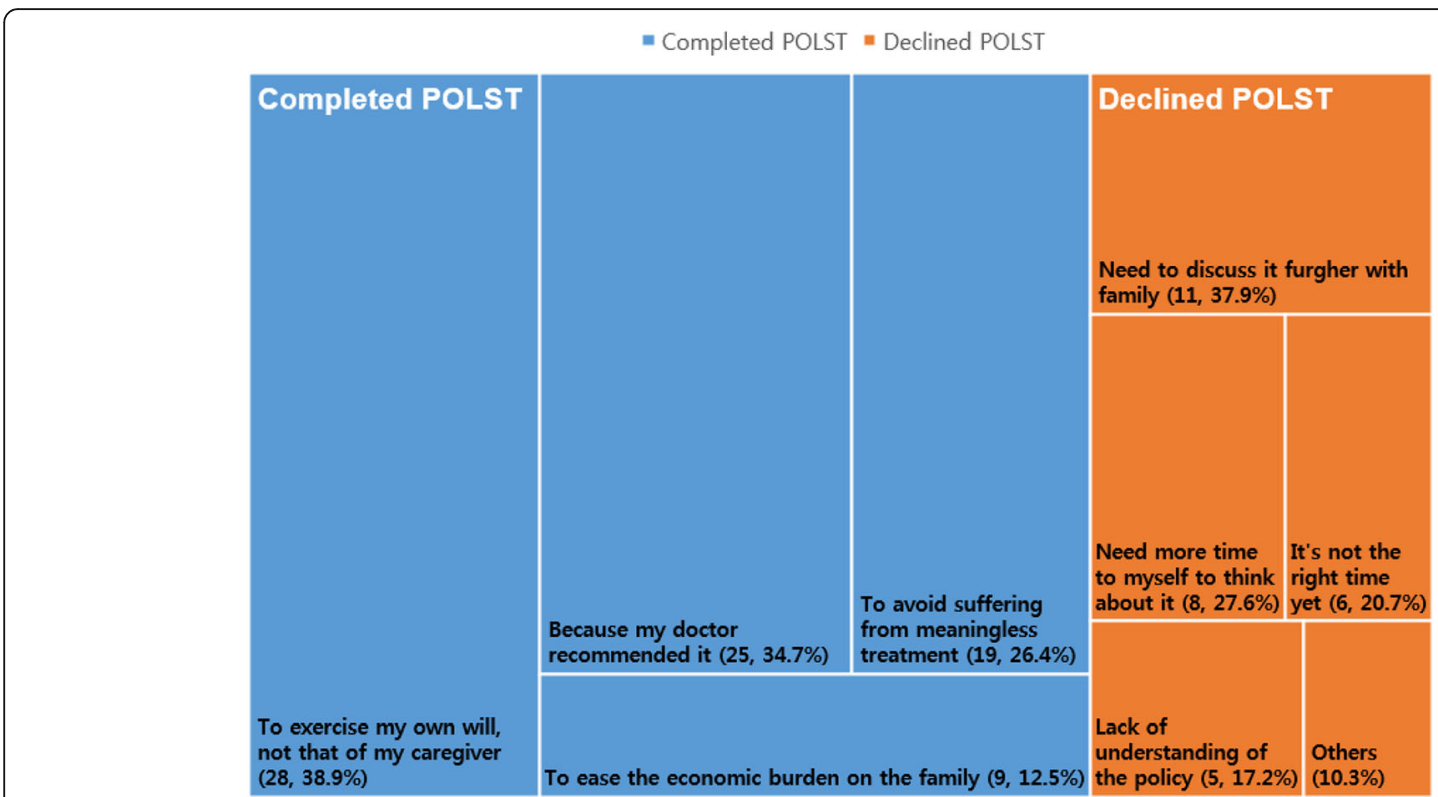

Fig. 2 POLST completion rate and reasons for completing or declining to complete POLST. The total POLST completion rate was 71.3\%. A larger area is indicative of a higher response rate. "To exercise my own will, not that of my caregiver" was the most popular reason for completing POLST, and "need to discuss it further with family" was the most common reason for declining POLST

Table 2 POLST completion rate according to demographic features

\begin{tabular}{|c|c|c|c|c|}
\hline Variable & $\begin{array}{l}\text { Total } \\
(\mathrm{N}=101)\end{array}$ & POLST completion & POLST non-completion & $p$-value \\
\hline Age, n. (\%) & & & & 0.826 \\
\hline 64 or lower & $52(51.5 \%)$ & $38(73.1 \%)$ & $14(26.9 \%)$ & \\
\hline 65 or above & $49(48.5 \%)$ & $34(69.4 \%)$ & $15(30.6 \%)$ & \\
\hline Gender, n. (\%) & & & & 1.000 \\
\hline Male & $54(53.5 \%)$ & $38(70.4 \%)$ & $16(29.6 \%)$ & \\
\hline Female & $47(46.5 \%)$ & $34(72.3 \%)$ & $13(27.7 \%)$ & \\
\hline Education, n. (\%) & & & & 0.007 \\
\hline High school or below & $39(38.6 \%)$ & $22(56.4 \%)$ & $17(43.6 \%)$ & \\
\hline High school or above & $61(60.4 \%)$ & $50(82.0 \%)$ & $11(18.0 \%)$ & \\
\hline Monthly income, n. (\%) & & & & 0.169 \\
\hline$\$ 2000$ or lower & $77(76.2 \%)$ & $51(66.2 \%)$ & $26(33.8 \%)$ & \\
\hline Over $\$ 2000$ & $20(19.8 \%)$ & $17(85.0 \%)$ & $3(15.0 \%)$ & \\
\hline Religion, No. (\%) & & & & 1.000 \\
\hline No religion & $43(42.6 \%)$ & $31(72.1 \%)$ & $12(27.9 \%)$ & \\
\hline Follow religion & $57(56.4 \%)$ & $40(70.2 \%)$ & $17(29.8 \%)$ & \\
\hline $\begin{array}{l}\text { Time from cancer diagnosis } \\
\text { to POLST suggestion, n. (\%) }\end{array}$ & & & & 0.172 \\
\hline One year or less & $20(19.8 \%)$ & $17(85.0 \%)$ & $3(15.0 \%)$ & \\
\hline More than one year & $81(80.2 \%)$ & $55(67.9 \%)$ & $29(35.8 \%)$ & \\
\hline $\begin{array}{l}\text { Follow-up period by physician } \\
\text { who suggested POLST, n. (\%) }\end{array}$ & & & & 0.826 \\
\hline One year or less & $40(39.6 \%)$ & $28(70.0 \%)$ & $12(30.0 \%)$ & \\
\hline More than one year & $61(60.4 \%)$ & $44(72.1 \%)$ & 17 (27.9\%) & \\
\hline
\end{tabular}


Table 3 POLST completion rate according to degree of understanding

\begin{tabular}{|c|c|c|c|c|}
\hline Variable & $\begin{array}{l}\text { Total } \\
(N=101)\end{array}$ & $\begin{array}{l}\text { POLST- completion } \\
(n=72)\end{array}$ & $\begin{array}{l}\text { POLST non-completion } \\
(n=29)\end{array}$ & $p$-value \\
\hline \multicolumn{5}{|l|}{$\begin{array}{l}\text { Familiarity with "life } \\
\text { sustaining treatment", n. (\%) }\end{array}$} \\
\hline Very familiar & $9(8.9 \%)$ & $7(9.7 \%)$ & $2(6.9 \%)$ & \\
\hline Familiar & $31(30.7 \%)$ & $26(36.1 \%)$ & $5(17.2 \%)$ & \\
\hline Fair understanding & $35(34.7 \%)$ & $27(37.5 \%)$ & $8(27.6 \%)$ & \\
\hline Not familiar & $15(14.9 \%)$ & $7(9.7 \%)$ & $8(27.6 \%)$ & \\
\hline Hardly know about it & 10 (9.9\%) & $5(6.9 \%)$ & $5(17.2 \%)$ & \\
\hline Unaware & $1(1.0 \%)$ & $0(0.0 \%)$ & $1(3.4 \%)$ & \\
\hline Median score ${ }^{a}$ & 3.14 & 3.32 & 2.68 & 0.016 \\
\hline \multicolumn{5}{|l|}{ Information sources ${ }^{b}$} \\
\hline Family or other people & $6(5.9 \%)$ & $6(8.3 \%)$ & $0(0.0 \%)$ & \\
\hline $\begin{array}{l}\text { Newspaper or } \\
\text { television news }\end{array}$ & $50(49.5 \%)$ & $36(50.0 \%)$ & $14(48.3 \%)$ & \\
\hline Internet & $7(6.9 \%)$ & $6(8.3 \%)$ & $1(3.4 \%)$ & \\
\hline Physician & $29(28.7 \%)$ & $23(31.9 \%)$ & $6(20.7 \%)$ & \\
\hline Never heard about it & $11(10.9 \%)$ & $6(8.3 \%)$ & $5(17.2 \%)$ & \\
\hline \multicolumn{5}{|l|}{$\begin{array}{l}\text { Familiarity with } \\
\text { "POLST", n. (\%) }\end{array}$} \\
\hline Very familiar & $11(10.9 \%)$ & $8(11.1 \%)$ & $3(10.3 \%)$ & \\
\hline Familiar & $22(21.8 \%)$ & $18(25.0 \%)$ & $4(13.8 \%)$ & \\
\hline Fair understanding & $26(25.7 \%)$ & $22(30.6 \%)$ & $4(13.8 \%)$ & \\
\hline Not familiar & $20(19.8 \%)$ & $13(18.1 \%)$ & $7(24.1 \%)$ & \\
\hline Hardly know about it & $22(21.8 \%)$ & $11(15.3 \%)$ & $11(37.9 \%)$ & \\
\hline Unaware & $0(0.0 \%)$ & $0(0.0 \%)$ & $0(0.0 \%)$ & \\
\hline Median score $\mathrm{a}^{\mathrm{a}}$ & 2.80 & 2.99 & 2.34 & 0.036 \\
\hline \multicolumn{5}{|l|}{ Information sources ${ }^{b}$} \\
\hline $\begin{array}{l}\text { Family or } \\
\text { other people }\end{array}$ & $7(6.9 \%)$ & $5(6.9 \%)$ & $2(6.9 \%)$ & \\
\hline $\begin{array}{l}\text { Newspaper or } \\
\text { television news }\end{array}$ & $38(37.6 \%)$ & $27(37.5 \%)$ & 11 (37.9\%) & \\
\hline Internet & $7(6.9 \%)$ & $6(8.3 \%)$ & $1(3.4 \%)$ & \\
\hline Physician & 40 (39.6\%) & $32(44.4 \%)$ & $8(27.6 \%)$ & \\
\hline Never heard about it & $17(16.8 \%)$ & $10(13.9 \%)$ & $7(24.1 \%)$ & \\
\hline
\end{tabular}

a Median score of awareness when "very familiar" was scored 5 and "never heard about it" was scored 1

${ }^{b}$ Multiple responses were allowed. All percentage data were presented with the percentage of cases

for each answer (Table 4). Interestingly, the answers recorded in the POLST documents and survey questionnaires were not perfectly concordant. When physicians asked patients whether or not they wanted to use hospice palliative care, 41 (56.9\%) out of 72 said "yes." However, when the participants were asked about this in a separate room during the questionnaire survey, seven out of 41 said they did not wish to use it. There were 21 (29.2\%) patients who completed POLST documentation but did not indicate their decision regarding hospice palliative care in the legal documents, and in the questionnaire, 13 of them indicated that they would not use a
Table 4 Preference regarding hospice palliative care

\begin{tabular}{llll}
\hline POLST & & \multicolumn{2}{l}{ Survey questionnaire } \\
\hline $\begin{array}{l}\text { Yes, I would like to } \\
\text { use hospice palliative } \\
\text { care, n. (\%) }\end{array}$ & $41(56.9 \%)$ & Yes & $34(47.2 \%)$ \\
$\begin{array}{l}\text { No, I would not like } \\
\text { to use hospice } \\
\text { palliative care, n. (\%) }\end{array}$ & $10(13.9 \%)$ & Yes & $7(9.7 \%)$ \\
Unanswered, n. (\%) & $21(29.2 \%)$ & Yes & $9(1.4 \%)$ \\
& & No & $13(1.1 \%)$ \\
\hline
\end{tabular}


hospice care center. According to the survey, the main reasons for using hospice palliative care were "to receive professional treatment for pain and symptoms" (26, $43.3 \%)$ and "to reduce the burden on caregivers" (25, 41.7\%). The most common reasons for not wanting to use hospice palliative care were "I don't think it would be particularly helpful" $(18,40.0 \%)$ and "because I want to be treated by the doctor I've been seeing" (17, 37.8\%).

\section{Discussion}

We investigated the POLST completion rate in patients with metastatic or recurrent cancer who were undergoing active anti-cancer therapy. When it was suggested by their physicians, $71.3 \%$ of patients agreed to prepare POLST, and most of them wanted to suspend CPR, artificial respiration, and hemodialysis. The POLSTcompletion group showed a higher proportion of highly educated patients and a better understanding of POLST. The participants answered that, typically, they would set up the EOL care plan of their own will. Their reasons for agreeing to or declining POLST were diverse.

More patients responded positively to POLST than expected by the researchers. To our knowledge, this is the first study that involved physicians' active recommendation of POLST. On the basis of the high rate of POLST completion, we can infer that patients with metastatic or recurrent cancer were ready to begin a conversation about EOL, even during active treatment. However, the high acceptance rate could have been due to the "initial effect" of the policy. As this is the very beginning of POLST legislation in South Korea, the number of patients who had experienced multiple lines of chemotherapy and had considered their EOL in depth would have accumulated over time.

In our study, $67.1 \%$ of who completed POLST wanted to maintain chemotherapy in their EOL, which could be a result of misunderstanding. The Korean POLST is a single-step decision for patients. Nevertheless, in implementing the document, doctors' decision involves two steps: initial time of POLST documentation and near EOL. Therefore, patients could be confused about the timing of POLST application. In our additional followup study, there were 35 of the total patients who wanted to maintain chemotherapy in their EOL were available to be asked again. After we had explained the situation thoroughly and asked whether they would still stand by their formal decision, 31 patients $(88.6 \%)$ revised to not receiving chemotherapy. Based on this phenomenon, we suggest to revise Korean POLST to two-step approach.

Previous investigations from East Asian countries have shown that the decision to discontinue life-sustaining treatment is dominated by patients' caregivers [4-7]. However, in our survey, most patients reported that they had made or would make their own decisions about
EOL care. This phenomenon might have been owing to the study protocol-we did not give any prior notice about POLST and required participants to make their decision on the same day. Even though the patients had no time to discuss it with their families, more than half willingly completed POLST documentation on the same day.

It was their spouses that patients most often chose to have the discussion with, and doctors were preferred over offspring or siblings. This is consistent with the results of previous studies [8-10]. Regarding the reason for preparing POLST, a significant proportion of the participants (34.7\%) answered that they did so at their doctor's recommendation. Further, the wish to be treated by a familiar doctor was one of the major reasons for not using a hospice palliative center (37.8\%). Therefore, the health care provider as well as familial support is important in patients' critical decision-making process and EOL care.

Debate over the self-determination of EOL care has been actively conducted in the West, and POLST and $\mathrm{AD}$ originated from the concept of the "living will" that was first proposed by the Euthanasia Society of America in 1967 [11]. With this, lawyers and medical staff in the United States tried to provide legal grounds for living wills at the state level. However, the controversy over the "right to die" continued, and social discussion around EOL care expanded as several historical events occurred $[12,13]$. In contrast to historical cases in the West, important cases in South Korea involved older subjects [14]. After several events and legal decisions, South Koreans started reviewing the social consensus on the following question: "What is 'dying with dignity'?

While the cases in the West mainly centered around the issue of "self-determination rights" of patients who entered a vegetative state at a younger age, the issues of the burden of support and financial difficulties of families have aroused more social sympathy in South Korea [14]. This is owing to differences in social values and cultural environment. Based on Confucianism, people in East Asian countries such as South Korea feel a high obligation to care for the elderly and provide familial support [15]. In many aspects, the care burden of the elderly has been reestablished as a problem to be solved by the household, not by social security services [16]. The pressure and stress created by the issues of who should support and who should be supported are considerably high in South Korea $[17,18]$. POLST and euthanasia can be a very dangerous scheme in this sociocultural environment. In some situations, someone may suspend his or her life-sustaining treatment to lessen the burden of the family. This kind of internal or external conflict can seriously undermine the original intent of POLST. In our study, $12.5 \%$ of the participants who agreed to complete POLST documentation said they decided to do so mainly because they wanted to 
ease the economic burden of their families. There was no significant difference when we compared the answers of the groups classified by monthly income, but further clarification is needed.

There has been some argument regarding when to prepare POLST in end-stage patients $[19,20]$. In our study, a large percentage of participants answered that it would be desirable to prepare ACP when they were still young and healthy. Caregivers who accompanied the patients during the survey also expressed agreement with the withdrawal of futile life-sustaining treatment and asked whether they could fill out the document too. Although POLST in South Korea is restricted to end-stage patients, it is noteworthy that people are now more aware of the need to prepare for "dying well."

The more patients understood life-sustaining treatment or POLST, the higher the completion rate was. A total of $66.7 \%$ of the completion group answered that they had more than a fair understanding of the system. Most of them got their information from traditional media such as the television or newspapers. A few weeks after the legislation, the South Korean POLST system began being promoted online. However, this is not the most effective way for older patients and those whose general condition is poor to access the relevant information. Physicians were the major source of information about EOL care to patients with cancer; thus, in-hospital education or counseling from medical staff could provide better assistance.

Our study had several limitations. First, the sample size was too small to achieve statistically significant $p$ values in many aspects. We also could not secure the diversity of cancer type because of the hospital system, making it difficult to represent the complete situation. Second, because different doctors recommended POLST in their own ways, the protocol could not be fully standardized. There was bias due to physicians' diverse experience and rapport with patients. We assume that all study participants were well aware of the policy, but there might be discordance between doctors regarding eligibility criteria, length of instruction, and understanding of the process. The extraordinarily high rate of patients continuing with chemotherapy $(67.1 \%)$ might be a result of misunderstanding. Third, because we did not collect detailed data about patients' income and medical expense sources, we could not fully investigate the specific role of financial problems in POLST decisionmaking. The information on family members who were present at the completion day is also lacking, making it difficult to account for familial factors.

From social consensus to medical practice, there are still many issues that need to be addressed. Improving structural support for terminally ill patients, developing hospitalbased assistance services, and providing education about the system would help yield positive outcomes. This study is meaningful in that it gathered opinions on the medical care of patients from the patients themselves, and not from their caregivers. We hope that the findings of this and subsequent studies can help people understand the POLST system and patients' real attitudes toward EOL.

\section{Conclusion}

With this study, we found that it is important to increase patients' understanding of the policy in establishing endof-life care. Better education and more efficient information sharing can be helpful in improving understanding of POLST in patients with cancer.

\section{Supplementary information}

Supplementary information accompanies this paper at https://doi.org/10 1186/s12904-019-0475-9.

Additional file 1: Table S1. Responses to questions about end-of-life care.

Additional file 2: Figure S1. The relationship of advance care planning $(A C P)$ with advance directives (AD) and Physician Orders for LifeSustaining Treatment (POLST).

Additional file 3. Awareness and attitudes of patients with cancer toward physician orders for life-sustaining treatment(POLST).

Abbreviations

ACP: Advance Care Plan; AD: Advance Directive; EOL: End of Life; POLST: Physician Orders for Life-Sustaining Treatment

\section{Acknowledgments}

The authors acknowledge the support of the members of the Korea University Anam Hospital Cancer Center.

\section{Authors' contributions}

JWK and KHP designed the study and wrote the manuscript. JYC, WJJ, and YJC assisted with data collection and analysis. YSC contributed to the final version of the manuscript. SWS and YHK helped patient enrollment and supervised the project with constructive opinion on data interpretation. All authors read and approved the final manuscript.

\section{Funding}

The authors received no financial support for the research, authorship, and/ or publication of this article.

\section{Availability of data and materials}

The datasets supporting the conclusions of this article are included within the article and its additional files.

\section{Ethics approval and consent to participate}

Written informed consent was obtained from all participants and the study was approved by the Institutional Review Board (IRB) of Korea University Hospital (IRB no. 2018AN0152).

\section{Consent for publication}

We received consent to use patients' data through a form approved by the IRB.

Competing interests

The authors declare that they have no competing interests.

\section{Author details}

${ }^{1}$ Division of Oncology/Hematology, Department of Internal medicine, Korea University Anam Hospital, 73 Goryeodae-ro Seongbuk-gu, Seoul 02841, South 
Korea. ${ }^{2}$ Department of Family Medicine, Korea University Guro Hospital, 97 Guro-dong Gil, Guro-gu, Seoul 08308, South Korea.

Received: 11 June 2019 Accepted: 2 October 2019

Published online: 22 October 2019

\section{References}

1. Sang-Min Lee M, Su-Jung Kim M, Youn Seon Choi M, Dae Seog Heo M, Bo Moon Choi M, Daekyun Kim M, et al. Consensus guidelines for the definition of the end stage of disease and last days of life and criteria for medical judgment. J Korean Med Assoc. 2018;61 (8):509-21.

2. Keam B, Yun YH, Heo DS, Park BW, Cho CH, Kim S, et al. The attitudes of Korean cancer patients, family caregivers, oncologists, and members of the general public toward advance directives. Support Care Cancer. 2013;21(5):1437-44.

3. McLaughlin LA, Braun KL. Asian and Pacific islander cultural values: considerations for health care decision making. Health Soc Work. 1998;23(2):116-26.

4. Alden DL, Friend J, Lee PY, Lee YK, Trevena L, Ng CJ, et al. Who decides: me or we? Family involvement in medical decision making in eastern and Western countries. Med Decis Mak. 2017;38(1):14-5.

5. Oh DY, Kim JH, Kim DW, Im SA, Kim TY, Heo DS, et al. CPR or DNR? End-oflife decision in Korean cancer patients: a single center's experience. Support Care Cancer. 2006;14(2):103-8.

6. Fujimoto K, Minami S, Yamamoto S, Ogata Y, Koba T, Futami S, et al. Comparison of timing and decision-makers of do-not-resuscitate orders between thoracic cancer and non-cancer respiratory disease patients dying in a Japanese acute care hospital. Support Care Cancer. 2014;22(6):1485-92.

7. Baek SK, Chang HJ, Byun JM, Han JJ, Heo DS. The association between end-of-life care and the time interval between provision of a do-not-resuscitate consent and death in cancer patients in Korea. Cancer Res Treat. 2017;49(2):502.

8. Kwon I, Koh Y, Yun Y, Heo D, Seo S, Kim H, et al. A study of the attitudes of patients, family members, and physicians toward the withdrawal of medical treatment for terminal patients in Korea. Korean J Med Ethics. 2010;13(1):1-16.

9. Glick HR. The right-to-die: state policymaking and the elderly. J Aging Stud. 1991;5(3):283-307.

10. US SC. Cruzan V. Director, Missouri Department of Health. West's Supreme Court reporter. 1990;110:2841.

11. Brown BA. The history of advance directives a literature review. J Gerontol Nurs. 2003;29(9):4-9.

12. Greco PJ, Schulman KA, Lavizzo-Mourey R, Hansen-Flaschen J. The patient self-determination act and the future of advance directives. Ann Intern Med. 1991;115(8):639-43.

13. Annas GJ. When procedures limit rights: from Quinlan to Conroy. Hast Cent Rep. 1985;15(2):24-6.

14. Ahn K, Bae H. Reflections on the movement for the legalization of death with dignity as withdrawal of futile life-sustaining treatment in South Korea. J Korean L. 2010;10:43.

15. Kim S, Cho B. A study of familism and family support for the aged. J Korean Home Manag Assoc. 1991;9(2):303-22.

16. Jacobs D. Low public expenditures on social welfare: do east Asian countries have a secret? Int J Soc Welf. 2000;9(1):2-16.

17. Knight BG, Robinson GS, Longmire CVF, Chun M, Nakao K, Kim JH. Cross cultural issues in caregiving for persons with dementia: do familism values reduce burden and distress? Ageing Int. 2002;27(3):70-94.

18. Rhee $\mathrm{KO}$, Lee MJ. A study on caregiving burden among family caregivers of impaired elderly. Journal of Korea Gerontological Society. 2000;20(2):215-28.

19. Sudore RL, Fried TR. Redefining the "planning" in advance care planning: preparing for end-of-life decision making. Ann Intern Med. 2010;153(4):256-61.

20. Emanuel LL, Danis M, Pearlman RA, Singer PA. Advance care planning as a process: structuring the discussions in practice. J Am Geriatr Soc. 1995;43(4):440-6.

\section{Publisher's Note}

Springer Nature remains neutral with regard to jurisdictional claims in published maps and institutional affiliations.

Ready to submit your research? Choose BMC and benefit from:

- fast, convenient online submission

- thorough peer review by experienced researchers in your field

- rapid publication on acceptance

- support for research data, including large and complex data types

- gold Open Access which fosters wider collaboration and increased citations

- maximum visibility for your research: over $100 \mathrm{M}$ website views per year

At BMC, research is always in progress.

Learn more biomedcentral.com/submissions 